\title{
Farmacovigilancia: ¿y si notificamos?
}

\author{
Tomás Tena Trincado ${ }^{1,6,7}$, Ana Rivera Bocanegra ${ }^{2,6,7}$, Ana Isabel Beas Morales ${ }^{3,6,7}$, Clara Alonso Larrocha ${ }^{4,6,7}$, \\ Estefanía Bravo Moreno ${ }^{5,6,7}$, Rosario Rodríguez Rodríguez ${ }^{5,6,7}$ \\ 1. Farmacéutico comunitario en Lepe. 2. Farmacéutica comunitaria en La Palma del Condado. 3. Farmacéutica comunitaria en Ayamonte. 4. Farmacéutica \\ en el CIM del Colegio Oficial de Farmacéuticos de Huelva. 5. Farmacéutica comunitaria en Huelva. 6. Grupo de Atención Farmacéutica del COF de \\ Huelva. 7. Asociación de Farmacéuticos Adjuntos de Huelva.
}

\section{PALABRAS CLAVE}

farmacovigilancia, tarjeta amarilla, notificación, reacciones adversas a medicamentos, farmacéuticos comunitarios

\section{KEYWORDS}

pharmacovigilance, yellow card, reporting, adverse drug reactions, community pharmacists

\section{RESUMEN}

Introducción: Farmacovigilancia es la actividad de salud pública que tiene como objetivo la identificación, cuantificación, evaluación y prevención de los riesgos del uso de los medicamentos una vez comercializados, permitiendo así el seguimiento de los posibles efectos adversos. Según las últimas estadísticas del Centro Andaluz de Farmacovigilancia las notificaciones por farmacéuticos solo suponen el $9 \%$ de las realizadas por los profesionales sanitarios. A la vista de estos datos se propuso un estudio para conocer los motivos de la falta de notificación y resolver los posibles problemas.

Método: Se elaboró un cuestionario ad hoc, que se envió a todos los farmacéuticos de la provincia de Huelva.

Resultados: Participaron 66 farmacéuticos. El 91\% conoce la tarjeta amarilla, dispone de ella el 45\%, conoce la vía web el 34\%, ha notificado alguna vez 34\% (7\% lo hace siempre que se encuentra una reacción adversa). El $60 \%$ no ha notificado nunca y un $15 \%$ piensa que no es obligatorio. Los motivos por los que no se notifica son falta de información $44 \%$, falta de formación $41 \%$, falta de tiempo 36\% y no disponer de tarjeta amarilla $29 \%$. Los titulares demandan una mayor información y formación. Los adjuntos ven la falta de tiempo como principal motivo de la no notificación.

Discusión: Ante la poca notificación por los farmacéuticos y la importancia de este tema, se propone una campaña de concienciación entre los farmacéuticos de Huelva para mejorar estos resultados. Para ello se enviará a todos los colegiados un díptico formativo con la finalidad de resolver los principales inconvenientes de la notificación.

\section{Pharmacovigilance: why not report?}

\section{ABSTRACT}

Introduction: Pharmacovigilance is the public health activity that identifies, quantifies, evaluates and prevents the risks of the use of medicines after their introduction upon the market, thus allowing follow-up of their possible adverse effects. According to the latest statistics of the Andalusian Pharmacovigilance Center, reports by pharmacists represent only $9 \%$ of all reports by health professionals. In view of these data, a study was designed to identify the reasons for this lack of reporting and resolve the possible problems.

Method: An ad hoc questionnaire was administered among all the pharmacists of the province of Huelva (Spain).

Results: Sixty-six pharmacists participated in the study. A full $91 \%$ were aware of the yellow card pharmacovigilance reporting system, 45\% were in possession of the card, 34\% knew of the system website, and 34\% had made use of the system for reporting (7\% claimed to do so each time they identified an adverse reaction). Sixty percent had never reported, and 15\% believed that reporting is not mandatory. The reasons for not reporting were lack of information ( $44 \%)$, lack of training ( $41 \%)$, lack of time ( $36 \%$ ) and non-availability of the yellow card $(29 \%)$. The pharmacy owners expressed a need for greater information and training. The pharmacy employees regarded a lack of time to be the main reason for not reporting adverse reactions.

Discussion: In view of the low reporting levels among pharmacists and the great importance of this subject, we recommend the introduction of an awareness-enhancing campaign among the pharmacists in Huelva in order to improve the results obtained. In this regard, all the pharmacists will receive an informative leaflet with the aim of solving the main inconveniences of reporting.

Comunicación presentada como póster en el V Congreso Nacional de Farmacéuticos Comunitarios SEFAC en Barcelona 2012 y seleccionado para comunicación oral.

Recibido: $27-10-2013$

Aceptado: 28-3-2014

Disponible online: 1-6-2014
Financiación: Ninguna ajena.

Conflicto de intereses: Los autores declaran no existir conflicto de interés en relación con el contenido del presente artículo.

Cite este artículo como: Tena T, Rivera A, Beas Al, Alonso C, Bravo E, Rodríguez R. Farmacovigilancia: ¿y si notificamos?. Farmacéuticos Comunitarios. 2014 Jun 01:6(2):48-52. doi:10.5672/FC.2173-9218.(2014/Vol6).002.07

Autor para correspondencia: Tomás Tena Trincado (tomastenatrincado@gmail.com).

ISSN 1885-8619 OSEFAC (Sociedad Española de Farmacia Comunitaria). Todos los derechos reservados. 


\section{Introducción}

La farmacovigilancia se define como la actividad de salud pública que tiene como objetivo la identificación, cuantificación, evaluación y prevención de los riesgos del uso de los medicamentos una vez comercializados, permitiendo así el seguimiento de los posibles efectos adversos de los medicamentos (1). Cuando un medicamento sale al mercado se han identificado efectos adversos muy frecuentes y frecuentes ya que se han probado en varios cientos de miles de pacientes en los ensayos previos, pero una vez comercializados el medicamento es utilizado por millones de pacientes y aquellos efectos adversos que son poco frecuentes pueden estar aún no identificados y producir daños irreparables, de ahí la importancia de la farmacovigilancia y la importancia que tiene que todos los profesionales sanitarios implicados con el medicamento participen en el sistema (2-4).

La Ley 29/2006 de 26 de julio consagra definitivamente la importancia de la farmacovigilancia como elemento esencial del medicamento y expresa: "En materia de farmacovigilancia, tanto de medicamentos de uso humano como de uso veterinario, el capítulo IV regula las actividades de salud pública tendentes a la identificación, cuantificación, evaluación, y prevención de los riesgos del uso de los medicamentos una vez comercializados, permitiendo así el seguimiento de sus posibles efectos adversos, siendo de destacar el sistema español de farmacovigilancia, en el que las administraciones sanitarias han de realizar lo necesario para recoger, elaborar y en su caso procesar toda la información útil para la supervisión de medicamentos y en particular la información sobre reacciones adversas a los mismos, así como para la realización de cuantos estudios se consideren necesarios para evaluar su seguridad" (3). En su artículo 53 queda claramente establecida la obligatoriedad de comunicar las sospechas de reacciones adversas por parte de los profesionales sanitarios: "Los profesionales sanitarios tienen el deber de comunicar con celeridad a los órganos competentes en materia de farmacovigilancia de cada comunidad autónoma las sospechas de reacciones adversas de las que tengan conocimiento y que pudieran haber sido causadas por medicamentos" (3).
El Real Decreto 1344/2007 de 11 de octubre sobre farmacovigilancia de medicamentos de uso humano, recuerda que los profesionales sanitarios son parte del sistema español de farmacovigilancia y, en su artículo 7 , enumera y describe las obligaciones de los profesionales sanitarios respecto a farmacovigilancia (1).

Aunque la tarjeta amarilla es un sistema de notificación que ya se venía utilizando con anterioridad, a partir del Real Decreto se establece que la notificación al sistema español de farmacovigilancia humana de una reacción adversa se hará mediante tarjeta amarilla al centro de la comunidad autónoma correspondiente (1,3-5)

Desde el punto de vista práctico, tiene un interés especial para los farmacéuticos el triángulo amarillo que identifica a los medicamentos con principios activos nuevos en España y que acompañará a estos durante los cinco años posteriores a su autorización (1).

Según las últimas estadísticas facilitadas por el Centro Andaluz de Farmacovigilancia, las notificaciones llevadas a cabo por los farmacéuticos de atención primaria y comunitarios solo suponen el 9\% del total de las notificaciones realizadas por los profesionales sanitarios (6), siendo este el profesional sanitario que debiera estar por su formación y proximidad a la población el más implicado en la farmacovigilancia. Ante esta situación nos preguntamos por qué el farmacéutico comunitario no participa en el sistema de farmacovigilancia.

El objetivo del trabajo fue valorar el grado de conocimiento sobre farmacovigilancia en farmacéuticos comunitarios onubenses e identificar los motivos de la falta de notificación con objeto de solventarlos y prevenirlos.

\section{Material y métodos}

Estudio descriptivo, transversal, utilizando un cuestionario sobre farmacovigilancia con preguntas que permiten identificar los objetivos del estudio (figura 1). Este cuestionario consta de dos bloques de preguntas: el primero evalúa el grado de conocimiento de la farmacovigilancia y el

\section{ENCUESTA DE FARMACOVIGILANCIA DIRIGIDA A FARMACÉUTICOS COMUNITARIOS}

Provincia farmacia: Municipio:

Fecha:

Farmacéutico: Titular $\square \quad$ Adjunt. $\square \quad$ Sustit. $\square$

$\begin{array}{lrrr}\text { Sabe qué es la farmacovigilancia: } & \text { Si } \square & \text { No } \square \\ \text { Conoce la tarjeta amarilla: } & \text { Si } \square & \text { No } \square & \\ \text { Dispone de ella en la farmacia: } & \text { Si } \square & \text { No } \square & \\ \text { Conoce la tarjeta amarilla vía web: } & \text { Si } \square & \text { No } \square & \\ \text { Ha notificado alguna vez: } & \text { Si } \square & \text { No } \square & \\ \text { Con qué frecuencia notifica: } & \text { Siempre } \square & \text { Nunca } \square & \text { A veces } \square \\ \text { Piensa que es obligatorio: } & \text { Si } \square & \text { No } \square & \end{array}$

En general hay muy baja notificación por parte de los farmacéuticos ¿Cuál es su caso?:
1. Falta de información
2. Falta de formación
3. No dispongo de tarjeta amarilla
4. Falta de tiempo
5. No me interesa este tema
6. Considero que no es obligatorio
7. La vía de comunicación no es la adecuada
8. No es relevante
9. Otros:

$\begin{array}{ll} & \square \\ & \square \\ & \square \\ & \square \\ & \square \\ & \square \\ & \square \\ & \\ & \square\end{array}$

Figura 1 Cuestionario utilizado para la encuesta 


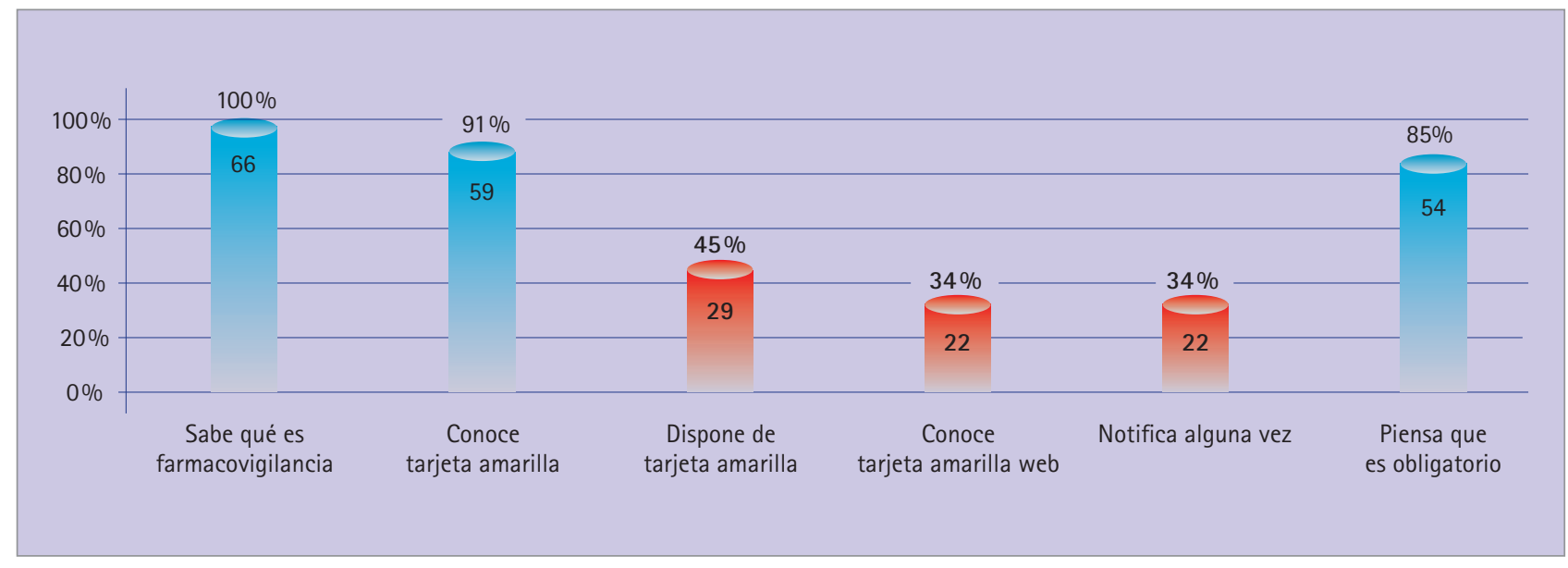

Figura 2 Datos generales de la encuesta de farmacovigilancia (en rojo los datos más preocupantes de la encuesta)

segundo evalúa los motivos de la falta de notificación. La distribución y recogida de los cuestionarios se centralizó en el Colegio Oficial de Farmacéuticos de Huelva y desde ahí se distribuyó a todos los farmacéuticos comunitarios de la provincia de Huelva, enviándose los cuestionarios dos veces, en marzo y mayo del 2012. Los farmacéuticos una vez que rellenaban el cuestionario volvían a enviar el cuestionario al Colegio Oficial por el mismo mecanismo obteniéndose datos de 66 farmacéuticos onubenses.

Los resultados fueron procesados $\mathrm{y}$ analizados mediante el programa estadístico SPSS $^{\circledR}$. Se utiliza como prueba estadística la chi cuadrado y se considera un nivel de confianza del $95 \%(p<0,05)$.

\section{Resultados}

Contestaros 66 farmacéuticos comunitarios que representan el $13 \%$ de los farmacéuticos comunitarios colegiados en la provincia de Huelva.

De los 66 farmacéuticos participantes en el estudio, 38 realizan su trabajo en el medio rural (57,6\%), 26 en medio urbano $(39,4 \%)$ y 2 no contestan $(3,0 \%) .35$ son titulares de farmacia $(56,5 \%), 24$ son adjuntos $(38,7 \%)$ y 7 sustitutos y regentes $(10,6 \%)$.

Los datos generales de la encuesta de conocimiento se presentan en la figura 2. Se señalan en rojo los ítems que obtuvieron menor resultado.

54 farmacéuticos piensan que es obligatorio $(84,4 \%)$, mientras que hay un 15,6\% de farmacéuticos que creen que notificar reacciones adversas no es obligatorio. Las frecuencias de notificación se muestran en la figura 3.

Se compararon todos estos resultados entre farmacéuticos rurales y urbanos, y entre farmacéuticos titulares y adjuntos (tabla 1)

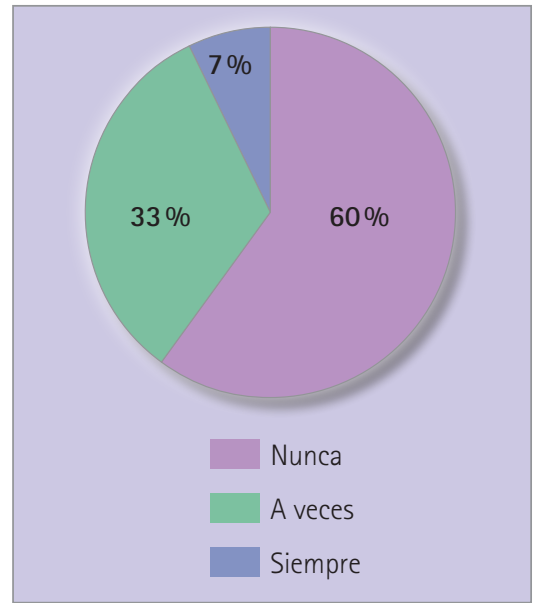

Figura 3 Frecuencia de notificación de los farmacéuticos en la provincia de Huelva

sin que se encontraran diferencias significativas en ninguna pregunta del primer bloque. En cuantos al segundo bloque de preguntas dirigidas a valorar los motivos de la no

Tabla 1 Distribución de las respuestas a las preguntas del primer bloque

\begin{tabular}{|l|c|c|c|c|c|c|c|c|c|c|c|c|}
\hline & \multicolumn{3}{|c|}{ Uural } & \multicolumn{3}{c|}{ Titular } & Adjunto \\
\cline { 2 - 6 } & Si & No & NC & Si & No & NC & Si & No & NC & Si & No \\
\hline
\end{tabular}




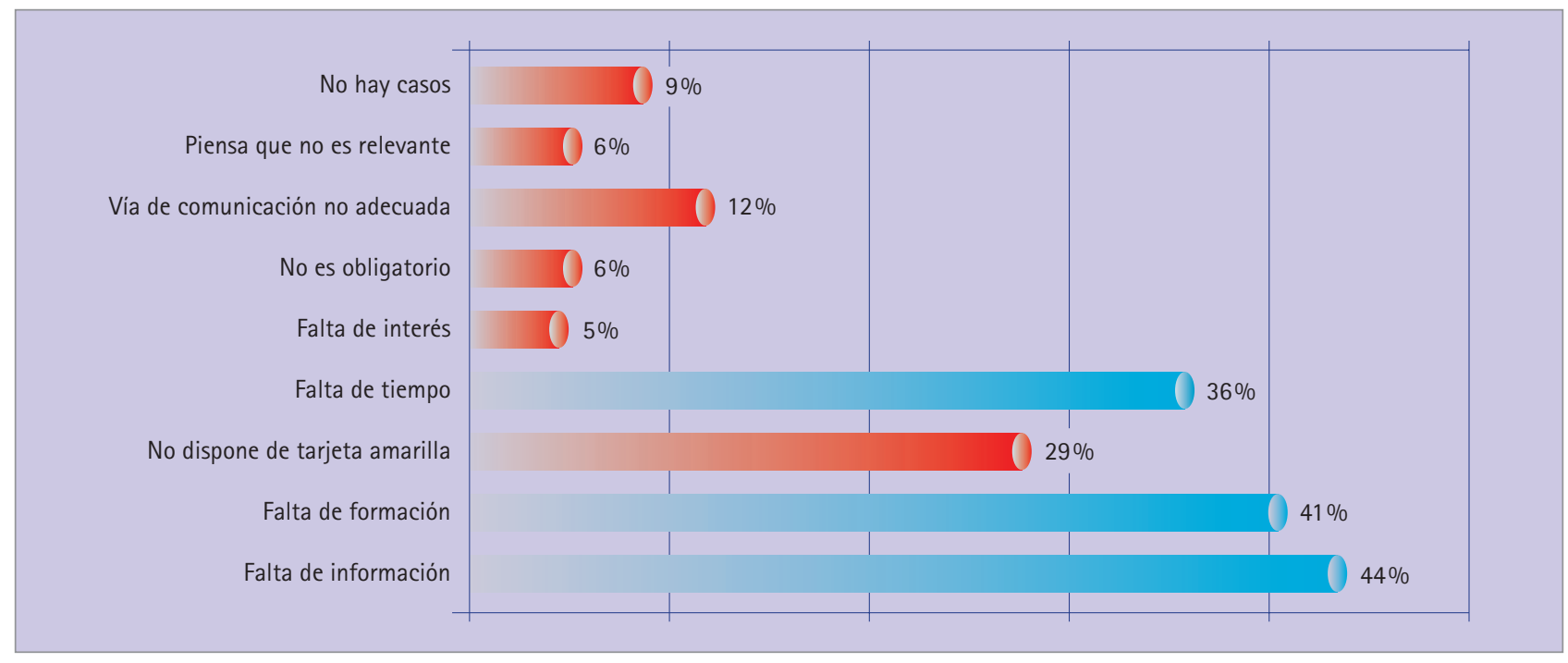

Figura 5 Motivos por los que los farmacéuticos de Huelva no notifican las reacciones adversas (en azul los principales motivos)

notificación, los resultados se presentan en la figura 5.

Se comparan igual que en el primer bloque diferencias entre farmacéuticos rurales y farmacéuticos urbanos sin encontrar diferencias significativas y también entra farmacéuticos titulares y adjuntos y aquí encontramos diferencias significativas en el motivo falta de tiempo en el que los adjuntos lo ven como principal motivo de no notificación: 22,9\% frente a 58,3\% $(p<0,05)$ (figura 6).

\section{Discusión}

Contamos en el estudio con la limitación de no utilizar un cuestionario validado y con un sesgo en la muestra, ya que solo habrán contestado los encuestados más predispuestos o con mayores conocimientos. El sistema elegido tampoco garantiza que los cuestionarios hayan llegado a todos los farmacéuticos comunitarios de la provincia. Aun así nos da una idea bastante clara de la actividad en materia de farmacovigilancia en la provincia de Huelva.

La tasa de respuesta es ligeramente inferior a la obtenida en otros estudios realizados en España (7).

En general y como indican las últimas estadísticas del Centro Andaluz de Farmacovigilancia de 2010 (6), los farmacéuticos comunitarios de la provincia de Huelva no participan en la notificación de reacciones adversas o lo hacen muy poco, aun cuando los farmacéuticos comunitarios son el colectivo que por su formación como expertos en el medicamento y proximidad al paciente debería de participar más activamente en este sistema. La mayoría conoce perfectamente qué es la farmacovigilancia y llama la atención que el 44\% no dispone de tarjeta amarilla aunque el Colegio Oficial las ha enviado al $100 \%$ de las farmacias de Huelva. Solo un $33 \%$ de farmacéuticos conoce la tarjeta amarilla vía web $\mathrm{y}$ además solo un $33 \%$ ha notificado alguna vez y solo un $7 \%$ notifica siempre que se encuentra alguna reacción adversa, que es la actuación correcta, y hay un 15\% de farmacéuticos que piensa que no es obligatorio. Esto nos demuestra el poco interés y la poca importancia que le dan los farmacéuticos comunitarios a la farmacovigilancia.

Los motivos principales de no notificación son: falta de información, falta de formación con un 40\% cada uno y falta de tiempo con un 36\%, destacando la diferencia que existe entre titulares y adjuntos en este apartado. Mientras los titulares opinan que no se notifica mayoritariamente por falta de formación y de información, los adjuntos achacan las carencias de

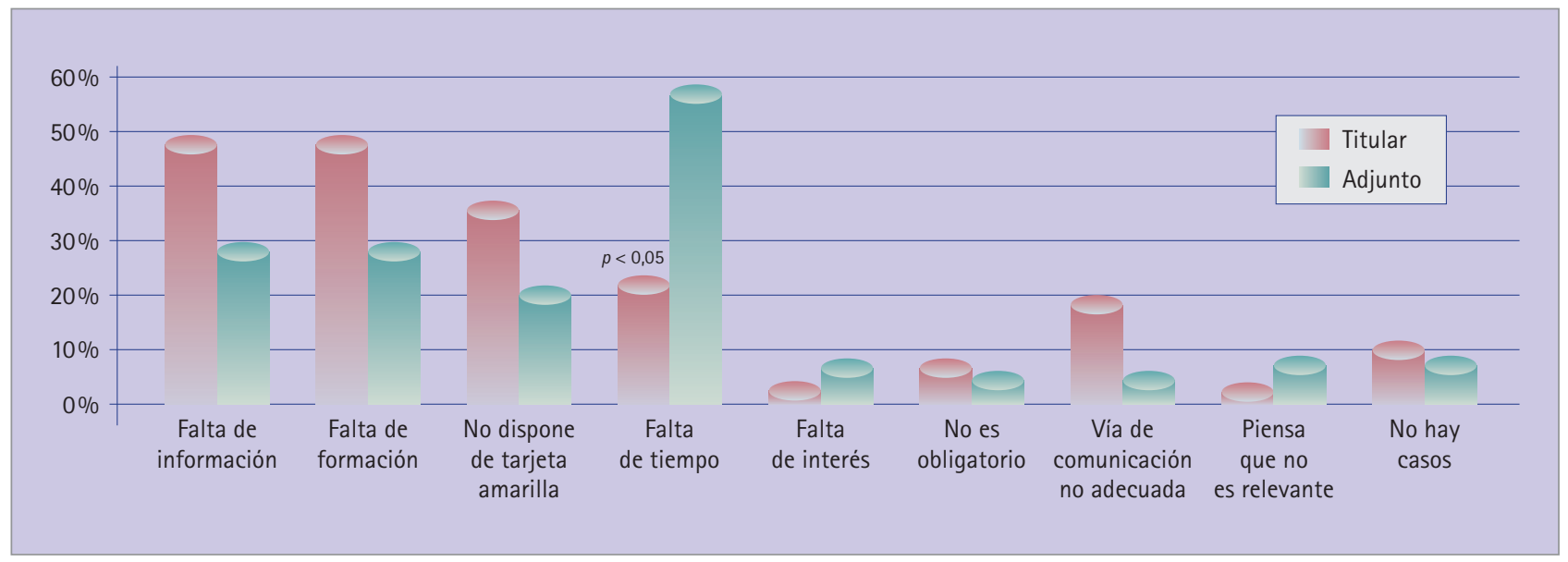

Figura 6 Motivos de la no notificación por parte de los farmacéuticos 
la farmacovigilancia en la farmacia comunitaria a la falta de tiempo.

Hoy en día el farmacéutico comunitario tiene suficiente formación e información, no olvidemos que es el experto en el medicamento, para detectar e identificar correctamente reacciones adversas a los medicamentos y conoce perfectamente el sistema nacional de farmacovigilancia para notificar correctamente. Con respecto a la falta de tiempo, con las nuevas tecnologías y la página web del Centro Andaluz de Farmacovigilancia, con la que se puede notificar en el mismo momento de identificar una reacción adversa y desde el mismo punto de venta de la farmacia comunitaria, tampoco es un motivo que no se pueda solventar $(5,8)$. Así que quizás sea un tema que esté un poco olvidado y para solucionarlo se va a poner en marcha una campaña de información a todos los farmacéuticos de la provincia de Huelva mediante díptico informativo y explicativo y posteriormente se repetirá la encuesta para reevaluar el estado de la farmacovigilancia y comprobar si nuestra actuación ha tenido un resultado positivo.

\section{Referencias bibliográficas}

1. Real Decreto 1344/2007, de 11 de octubre, por el que se regula la farmacovigilancia de medicamentos de uso humano. (BOE núm. 262, de 1 noviembre [rcl 2007, 1982])

2. Real Decreto $1345 / 2007$, de 11 de octubre, por el que se regula el procedimiento de autorización, registro y condiciones de dispensación de los medicamentos de uso humano fabricados industrialmente.

3. Ley 29/2006, de 26 de julio, de garantías y uso racional de los medicamentos y productos Sanitarios.

4. Ley 44/2003, de 21 de noviembre, de ordenación de las profesiones sanitarias.
5. Centro Andaluz de Farmacovigilancia [Internet]. Guía para la notificación espontánea de reacciones adversas 2007. [Acceso 10/09/2013]. Disponible en: http://www.juntadeandalucia.es/salud/servicios/contenidos/ farmacovigilancia/up/guia_notificadorCTAFV_noviembre2007versio2.pdf

6. Centro Andaluz de Farmacovigilancia [Internet]. Notificaciones por profesionales sanitarios 2011. [Acceso 10/09/2013]. Disponible en: http:// si.easp.es/csalud/cafv2011/profesionales_sanitarios.html

7. Acuña Ferradanes A, Gonzalez Añón D, Castillo Páramo A, Fornos Pérez JA, Andrés Iglesias JC, Andrés Rodríguez NF. Metodología para evaluar las actitudes y aptitudes sobre farmacovigilancia en los farmacéuticos comunitarios. Aplicación en la provincia de Pontevedra. Pharm Care Esp 2012; 14(3): 110-21.

8. Orden de 1 de marzo de 2000 , por la que se regulan los órganos encargados de la farmacovigilancia en la comunidad autónoma de Andalucía. 